\section{"AS-Group" \\ Neues Portal bietet Informationen rund um das Thema Active Surveillance}

\author{
- Bei Prostatakarzinomen mit niedrigem \\ Progressionsrisiko sollte vor allem die aktive \\ Überwachung, Active Surveillance, neben \\ Operation und Bestrahlung als Therapie- \\ strategie angeboten werden. Hier wird eine \\ Behandlung so lange zurückgestellt, bis sich \\ Hinweise auf eine Progression ergeben. Als \\ Kriterien für die Active Surveillance gelten \\ allgemein $\mathrm{CT} \leq 2$ (alle T2), PSA-Wert $\leq 10 \mathrm{ng} /$ \\ $\mathrm{ml}$, Gleason-Score $\leq 6$, außerdem $\leq 2$ posi- \\ tive Zylinder. Einige Zentren beziehungs- \\ weise Studien berücksichtigen auch die \\ PSA-Dichte $<0,2 \mathrm{ng} / \mathrm{ml} / \mathrm{ccm}$ als Einschluss-
}

kriterium. Dass Active Surveillance unter Alltagsbedingungen praktiziert werden kann, zeigt die HAROW-Studie, deren vorläufigen Ergebnisse im Frühjahr 2014 publiziert werden.

Active Surveillance hat als Therapiestrategie ihre Berechtigung; sie schützt vor den Auswirkungen einer Übertherapie. Trotzdem wird sie jedoch in der bisherigen Versorgungsrealität kaum berücksichtigt. Die Gründe dafür sind vielfältig und reichen von unzureichender Information (möglicherweise auch des Fachkreises, aber sicher der Öffentlichkeit), Ängsten (Patienten möchten möglichst schnell tumorfrei sein) bis hin zu einer nicht adäquaten Vergütung. Die Stiftung Männergesundheit hat einige dieser Lücken erkannt und bietet zum Thema Active Surveillance die Plattform www.as-beiprostatakrebs.de an. Sie wird zum DGUKongress am 25. September online gehen und umfassende Informationen zur Active Surveillance für Laien- und Fachkreise zur Verfügung stellen. Darüber hinaus können Betroffene in einem passwort-geschützen Forum ("AS-Group") ihre Erfahrungen austauschen und Gleichgesinnte finden. Weitere Schritte (Initialisierung von Selbsthilfegruppe, Erstellung eines Patientenaufklärungsbogens) sind geplant.

Bettina Albers

\section{Innovationspreis}

\section{Jetzt mit pfiffigen Ideen bewerben}

Cleverness wird belohnt. Ärzte, die mit innovativen Ansätzen ihren Praxiserfolg oder die Versorgung verbessert haben, können sich für den Innovationspreis 2013 bewerben. Es winken wertvolle Preise.

— Der Wettbewerb in der ambulanten wie stationären Versorgung verändert sich dramatisch. Dort wo die Versorgungsdichte hoch ist, haben es Einzelkämpfer und selbst größere Praxiseinheiten, die sich auf die traditionelle Patientenversorgung konzentrieren, immer schwerer, am Markt zu bestehen. In Gebieten, in denen Ärzte knapp werden, können die Praxen kaum den Ansturm der Patienten bewältigen. In beiden Fällen sind kreative Köpfe gefragt, die innovative Ansätze in der Patientenversorgung verfolgen, Ansätze, die nicht nur die Versorgungsqualität steigern, sondern sich auch finanziell für die Praxis lohnen.

Gemeinsam mit dem Biopharmaunternehmen UCB sucht die Verlagsgruppe Springer Medizin, zu der auch die "Ärzte Zeitung" und die URO-NEWS gehören, in diesem Jahr zum dritten Mal pfiffige Konzepte - mit dem Wettbewerb "Die innovative Arztpraxis" 2013. Teilnehmenden Praxen winken wertvolle Gewinne. Zudem werden die besten Ideen in der "Ärzte Zeitung“ vorgestellt.

\section{Ein Preis für zupackende Pioniere}

Der Innovationspreis wird unter anderem für zupackende Energie verliehen, mit der Praxisteams neue Konzepte im Gesundheitsmarkt erfolgreich umsetzen, so Steffen Fritzsche, Leiter Unternehmenskommunikation bei UCB. „Auch für den Innovationspreis 2013 suchen wir wieder kreative Ärzte, die die Herausforderung annehmen, innovative Medizin zu betreiben und die Versorgung zu

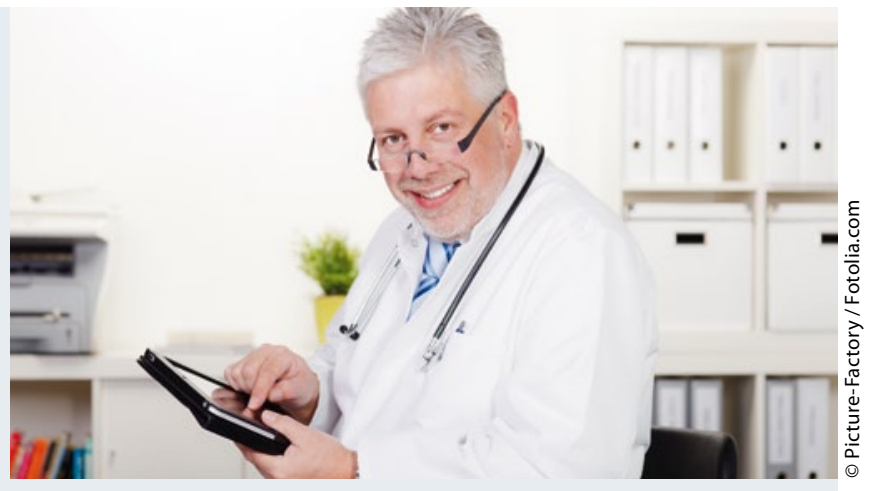

Konzept mit Esprit? Die Jury wird es begutachten.

verbessern", erläutert Fritzsche. Die Preisträger werden wie in den Vorjahren von einer unabhängigen Jury und von Internetnutzern auf "Ärzte Zeitung online“ bestimmt. Bewerben können sich Praxisteams online (s. u.).

\section{So können Sie teilnehmen}

Haben Sie eine innovative Idee, die Sie in Ihrer Praxis umsetzen wollen oder bereits umgesetzt haben? Dann beschreiben Sie bis zum 31. Oktober in unserem Online-Formular Ihre Idee und die Umsetzung. Dabei geht es auch darum, dass Sie zeigen, was Ihre Idee innovativ macht - zum Beispiel für die Versorgung von Patienten oder auch für die Wirtschaftlichkeit Ihrer Praxis. Sie können im Internet auch Dokumente hochladen, zum Beispiel Bilder oder Word-Dateien. Ihre Daten werden nur zur Ermittlung der Gewinner verwendet und nicht an Dritte weitergeleitet. Die Gewinnübergabe findet im Dezember statt. Matthias Wallenfels

Bewerbungsformular online unter www.aerztezeitung.de/extras/innovationspreis 